\title{
Two infinite integrals
}

By G. N. Watson.

(Received 22nd February, 1938. Read 4th March, 1938.)

The infinite integrals

$$
\begin{aligned}
& F(\lambda, \theta) \equiv \frac{1}{2 \pi} \int_{0}^{\infty} e^{i \lambda \cosh t} \frac{\sin \theta}{\cosh t+\cos \theta} d t, \\
& G(\lambda, \theta) \equiv \frac{1}{\pi} \int_{0}^{\infty} e^{i \lambda \cosh t} \frac{\cos \frac{1}{2} \theta \cosh \frac{1}{2} t}{\cosh t+\cos \theta} d t,
\end{aligned}
$$

in which $\lambda$ is positive and $-\pi<\theta<\pi$ were encountered by Kottler ${ }^{1}$ in a problem in the theory of diffraction. They have more recently been studied by Copson and Ferrar ${ }^{2}$, who obtained the remarkably simple Fourier series

$$
F(\lambda, \theta)=\frac{1}{2 i} \sum_{n=1}^{\infty} e^{-\frac{i n}{2} n i} h_{n}^{(1)}(\lambda) \sin n \theta,
$$

in which $h_{n}^{(1)}(\lambda)$ denotes a "cut Bessel function" of the third kind; this expansion is valid when $-\frac{1}{2} \pi<\theta<\frac{1}{2} \pi$; when $\frac{1}{2} \pi<|\theta|<\pi$, the term $\frac{1}{2} e^{-i \lambda \cos \theta} \operatorname{sgn} \theta$ has to be added to the expansion on the right.

This series is very elegant, but very slowly convergent, since, for fixed $\lambda$ and large $n$, we have

$$
h_{n}^{(1)}(\lambda)=\frac{2}{n \pi i} e^{-\frac{i}{2} n \pi i} \cos \frac{1}{2} n \pi+O\left(\frac{1}{n^{2}}\right) .
$$

Expansions better adapted for computation can be found ${ }^{3}$.

J. Kottler, Ann. der Physik, 71 (1923), 457-508.

2 E. T. Copson and W. L. Ferrar, these Proceedings (2), 5 (1938), 159-168.

${ }^{3}$ (Added in proof, 15th August, 1938.) I take this opportunity of making two remarks : (i) Prof. Copson has pointed out to me that, in the actual physical problem discussed by Kottler, no more is required than the approximate behaviour of $F(\lambda, \theta)$ and of $G(\lambda, \theta)$ when $\lambda$ is small and also when $\lambda$ is large ; I must, therefore, admit that, for the purposes of this problem, the Copson-Ferrar formulae are completely adequate and my own formulae contain superfluous and irrelevant information. (ii) Mr Ferrar has informed me that Dr Artur Erdélyi has written to him to point out that it is easy to derive a finite integral representing $F(\lambda, \theta)$ by means of Kottler's differential equation, and hence to obtain the expansion of $F(\lambda, \theta)$ for small values of $\lambda$. 
The clue to the problem of obtaining such expansions is the initial use of expansions which are power series in $\sin \frac{1}{2} \theta$; it will appear that such expansions are convergent when $\sin ^{2} \frac{1}{2} \theta<1$, i.e. when $\theta$ is not an odd multiple of $\pi$; moreover, these expansions can be modified so as to exhibit the behaviour of $F(\lambda, \theta)$ and $G(\lambda, \theta)$ when $\theta$ approaches these exceptional values.

It is evident that

$\frac{2}{\cosh t+\cos \theta}=\frac{1}{\cosh ^{2} \frac{1}{2} t-\sin ^{2} \frac{1}{2} \theta}=\sum_{n=0}^{N} \frac{\sin ^{2 n} \frac{1}{2} \theta}{\cosh ^{2 n+2} \frac{1}{2} t}+\frac{\sin ^{2 N+2 \frac{1}{2} \theta}}{\cosh ^{2 N+2} \frac{1}{2} t\left(\cosh ^{2} \frac{1}{2} t-\sin ^{2} \frac{1}{2} \theta\right)}$, and so

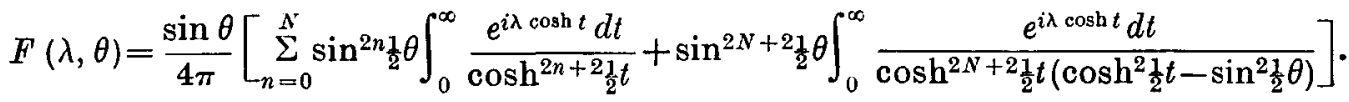

Now

$$
\begin{aligned}
& \left|\sin ^{2 N+2 \frac{1}{2} \theta} \int_{0}^{\infty} \frac{e^{i \lambda \cosh t} d t}{\cosh ^{2 N+2 \frac{1}{2} t}\left(\cosh ^{2} \frac{1}{2} t-\sin ^{2} \frac{1}{2} \theta\right)}\right| \leqq \sin ^{2 N+2 \frac{1}{2} \theta} \int_{0}^{\infty} \frac{d t}{\cosh ^{2} \frac{1}{2} t\left(1-\sin ^{2} \frac{1}{2} \theta\right)} \\
& =\frac{2 \sin ^{2 N+2} \frac{1}{2} \theta}{\cos ^{2} \frac{1}{2} \theta} \rightarrow 0,
\end{aligned}
$$

as $N \rightarrow \infty$ provided that $\theta$ is not an odd multiple of $\pi$; we have thus proved that

$$
F(\lambda, \theta)=\frac{\cos \frac{1}{2} \theta}{2 \pi} \sum_{n=0}^{\infty} \sin ^{2 n+1} \frac{1}{2} \theta \int_{0}^{\infty} \frac{e^{i \lambda \cosh t} d t}{\cosh ^{2 n+2 \frac{1}{2} t}},
$$

and, in a similar manner, we can prove that

$$
G(\lambda, \theta)=\frac{\cos \frac{1}{2} \theta}{2 \pi} \sum_{n=0}^{\infty} \sin ^{2 n} \frac{1}{2} \theta \int_{0}^{\infty} \frac{e^{i \backslash \cosh t} d t}{\cosh ^{2 n+1} \frac{1}{2} t}
$$

in the same circumstances.

We shall write these expansions in the forms

where

$$
\begin{aligned}
& F(\lambda, \theta)=\frac{1}{4} \cos \frac{1}{2} \theta \sum_{n=0}^{\infty} f_{n}(\lambda) \sin ^{2 n+1} \frac{1}{2} \theta, \\
& G(\lambda, \theta)=\frac{1}{4} \cos \frac{1}{2} \theta \sum_{n=0}^{\infty} g_{n}(\lambda) \sin ^{2 n} \frac{1}{2} \theta,
\end{aligned}
$$

$$
f_{n}(\lambda)=\frac{2}{\pi} \int_{0}^{\infty} \frac{e^{i \lambda \cosh t} d t}{\cosh ^{2 n+2 \frac{1}{2} t}}, \quad g_{n}(\lambda)=\frac{2}{\pi} \int_{0}^{\infty} \frac{e^{i \lambda \cosh t} d t}{\cosh ^{2 n+1} \frac{1}{2} t}
$$

and, after remarking that

$$
\begin{aligned}
& \left|f_{n}(\lambda)\right| \leqq \frac{2}{\pi} \int_{0}^{\infty} \frac{d t}{\cosh ^{2} \frac{1}{2} t}=\frac{4}{\pi}, \quad(n=0,1,2, \ldots), \\
& \left|g_{n}(\lambda)\right| \leqq{ }_{\pi}^{2} \int_{0}^{\infty} \frac{d t}{\cosh \frac{1}{2} t}=2, \quad(n=0,1,2, \ldots),
\end{aligned}
$$


we proceed to obtain expansions for $f_{n}(\lambda)$ and $g_{n}(\lambda)$ in the form of ascending series of powers of $\lambda$ and $\log \lambda$.

We begin by taking the formula ${ }^{1}$

$$
e^{i \lambda}\left\{i J_{0}(\lambda)-Y_{0}(\lambda)\right\}=\frac{2}{\pi} \int_{0}^{\infty} \exp \left(2 i \lambda \cosh _{2}^{2} t\right) d t
$$

and integrating it $n+1$ times from 0 to $\lambda$; we indicate this operation by the symbol

$$
\left[\int_{0}^{\lambda} d \lambda\right]^{n+1}
$$

There is no substantial difficulty in justifying the inversion of the order of the operations of passage to the limit and $(n+1)$-ple integration in the following analysis, in which Kummer's second formula ${ }^{2}$

$$
e^{i \lambda} J_{\nu}(\lambda)=\frac{\left(\frac{1}{2} \lambda\right)^{\nu}}{\Gamma(\nu+1)}{ }_{1} F_{1}\left(\nu+\frac{1}{2} ; 2 \nu+1 ; 2 i \lambda\right)
$$

has been used to simplify the integrand:

$$
\begin{aligned}
& {\left[\int_{0}^{\lambda} d \lambda\right]^{n+1} e^{i \lambda}\left\{i J_{0}(\lambda)-Y_{0}(\lambda)\right\}} \\
& =\left[\int_{0}^{\lambda} d \lambda\right]^{n+1} e^{i \lambda} \lim _{\nu \rightarrow 0}\left(\frac{1}{2} \lambda\right)^{\nu}\left\{i J_{\nu}(\lambda)-Y_{\nu}(\lambda)\right\} \\
& =\lim _{\nu \rightarrow 0}\left[\int_{0}^{\lambda} d \lambda\right]^{n+1} e^{i \lambda}\left(\frac{1}{2} \lambda\right)^{\nu} \frac{J_{-\nu}(\lambda)-e^{-\nu \pi i} J_{\nu}(\lambda)}{\sin \nu \pi} \\
& =\lim _{\nu \rightarrow 0}\left[\int_{0}^{\lambda} d \lambda\right]^{n+1} \frac{1}{\sin \nu \pi}\left\{\frac{1}{\Gamma(1-\nu)}{ }_{1} F_{1}\left(\frac{1}{2}-\nu ; 1-2 \nu ; 2 i \lambda\right)\right. \\
& \left.-\frac{e^{-\nu \pi i}\left(\frac{1}{2} \lambda\right)^{2 \nu}}{\Gamma(\nu+1)}{ }_{1} F_{1}\left(\nu+\frac{1}{2} ; 2 \nu+1 ; 2 i \lambda\right)\right\} \\
& =\lim _{\nu \rightarrow 0} \frac{\lambda^{n+1}}{\sin \nu \pi}\left\{\frac{1}{(n+1) ! \Gamma(1-\nu)}{ }_{2} F_{2}\left(\frac{1}{2}-\nu, 1 ; 1-2 \nu, n+2 ; 2 i \lambda\right)\right. \\
& -\frac{e^{-\nu \pi i}\left(\frac{1}{2} \lambda\right)^{2 \nu} \Gamma(2 \nu+1)}{\Gamma(\nu+1) \Gamma(2 \nu+n+2)}{ }_{1} F_{1}\left(\nu+\frac{1}{2} ; 2 \nu+n+2 ; 2 i \lambda\right) \\
& =\lim _{\nu \rightarrow 0} \frac{2^{-2 v} \lambda^{n+1}}{\Gamma\left(\frac{1}{2}\right) \sin \nu \pi}\left\{\sum_{m=0}^{\infty} \frac{\Gamma\left(\frac{1}{2}-\nu+m\right)(2 i \lambda)^{m}}{(n+m+1) ! \Gamma(1-2 \nu+m)}\right. \\
& \left.-\sum_{m=0}^{\infty} \begin{array}{c}
e^{-\nu \pi i} \Gamma\left(\nu+\frac{1}{2}+m\right)(2 \lambda)^{2 \nu}(2 i \lambda)^{2 m} \\
m ! \Gamma(2 \nu+n+m+2)
\end{array}\right\} .
\end{aligned}
$$

1 See my Theory of Bessel functions (Cambridge, 1922), 170.

2 See my Theory of Bessel functions, 191. 
We now calculate this limit by l'Hospital's rule in the usual way, by differentiating the denominator $\sin v \pi$ and differentiating the rest of the expression with respect to $\nu$, and then putting $\nu=0$; the result of this process is

$$
\begin{gathered}
\begin{array}{c}
\frac{\lambda^{n+1}}{\sqrt{ } \pi^{3}} \sum_{m=0}^{\infty} \frac{\Gamma\left(m+\frac{1}{2}\right)(2 i \lambda)^{m}}{m !(n+m+1) !}\left\{2 \psi(m+1)-2 \psi\left(m+\frac{1}{2}\right)+2 \psi(n+m+2)\right. \\
\quad+\pi i-2 \log 2 \lambda\}
\end{array} \\
=\frac{1}{(2 i)^{n+1} \sqrt{ } \pi^{3}} \sum_{m=n+1}^{\infty} \frac{\Gamma\left(m-n-\frac{1}{2}\right)(2 i \lambda)^{m}}{m !(m-n-1) !}\{4 \psi(m-n)-4 \psi(2 m-2 n) \\
\left.\quad+2 \psi(m+1)+\pi i-2 \log \frac{1}{2} \lambda\right\}
\end{gathered}
$$

and so we have proved that

$$
\begin{aligned}
& (2 i)^{n+1}\left[\int_{0}^{\lambda} d \lambda\right]^{n+1} e^{i \lambda}\left\{i J_{0}(\lambda)-Y_{0}(\lambda)\right\} \\
& =\frac{1}{\sqrt{ } \pi^{3}} \sum_{m=n+1}^{\infty} \frac{\Gamma\left(m-n-\frac{1}{2}\right)(2 i \lambda)^{m}}{m !(m-n-1) !}\{4 \psi(m-n)-4 \psi(2 m-2 n)+2 \psi(m+1) \\
& \left.\quad+\pi i-2 \log \frac{1}{2} \lambda\right\} .
\end{aligned}
$$

We now consider

$$
(2 i)^{n+1}\left[\int_{0}^{\lambda} d \lambda\right]^{n+1} \frac{2}{\pi} \int_{0}^{\infty} \exp \left(2 i \lambda \cosh ^{2} \frac{1}{2} t\right) d t
$$

and we evaluate it by carrying out the integrations with respect to $\lambda$ under the $t$-integral sign; the first of these inversions of order of integration is the only one which requires any but the simplest test to justify it, since the original integral (unlike the subsequent integrals) is not absolutely convergent, and, in fact, is not even convergent when $\lambda=0$; the actual details of justifying this inversion are left to the reader. We thus get

$$
\begin{aligned}
(2 i)^{n+1} & {\left[\int_{0}^{\lambda} d \lambda\right]^{n+1} \frac{2}{\pi} \int_{0}^{\infty} \exp \left(2 i \lambda \cosh ^{2} \frac{1}{2} t\right) d t } \\
& =\frac{2}{\pi} \int_{0}^{\infty} \frac{1}{\cosh ^{2 n+2 \frac{1}{2} t}}\left\{\exp \left(2 i \lambda \cosh ^{2} \frac{1}{2} t\right)-\sum_{m=0}^{n} \frac{\left.(2 i \lambda)^{m} \cosh ^{2 m \frac{1}{2}} t\right)}{m !}\right\} d t \\
& =e^{i \lambda} f_{n}(\lambda)-\frac{2}{\sqrt{ } \pi} \sum_{m=0}^{n} \frac{(n-m) !(2 i \lambda)^{m}}{m ! \Gamma\left(n-m+\frac{3}{2}\right)} .
\end{aligned}
$$

By comparing the two expressions which have been obtained for

$$
(2 i)^{n+1}\left[\int_{0}^{\lambda} d \lambda\right]^{n+1} e^{i \lambda}\left\{i J_{0}(\lambda)-Y_{0}(\lambda)\right\},
$$

we now infer that ${ }^{1}$

1 The labour of computing $f_{n}(\lambda)$ by means of this expansion is comparable with the labour of computing Bessel functions of the second kind. 


$$
\begin{aligned}
f_{u}(\lambda) & =\frac{2 e^{-i \lambda}}{\sqrt{ } \pi} \sum_{m=0}^{n} \frac{(n-m) !(2 i \lambda)^{m}}{m ! \Gamma\left(n-m+\frac{3}{2}\right)} \\
+ & \frac{2 e^{-i \lambda}}{\sqrt{ } \pi^{3}} \sum_{m=n+1}^{\infty} \frac{\Gamma\left(m-n-\frac{1}{2}\right)(2 i \lambda)^{m}}{m !(m-n-1) !}\{2 \psi(m-n)-2 \psi(2 m-2 n) \\
& \left.+\psi(m+1)+\frac{1}{2} \pi i-\log \frac{1}{2} \lambda\right\},
\end{aligned}
$$

and so we have obtained an expansion for $F(\lambda, \theta)$ in the somewhat complicated form

$$
\begin{gathered}
F(\lambda, \theta)=\frac{e^{-i \lambda} \cos \frac{1}{2} \theta}{2 \sqrt{ } \pi} \sum_{n=0}^{\infty} \sin ^{2 n+1} \frac{1}{2} \theta\left[\sum_{m=0}^{n} \frac{(n-m) !(2 i \lambda)^{m}}{m ! \Gamma(n-m+3)}\right. \\
+\frac{1}{\pi} \sum_{m=n+1}^{\infty} \frac{\Gamma\left(m-n-\frac{1}{2}\right)(2 i \lambda)^{m}}{m !(m-n-1) !}\{2 \psi(m-n)-2 \psi(2 m-2 n) \\
\left.\left.+\psi(m+1)+\frac{1}{2} \pi i-\log \frac{1}{2} \lambda\right\}\right] .
\end{gathered}
$$

When we interchange the order of the summations in this repeated series, we get

$$
\begin{aligned}
F(\lambda, \theta)= & \frac{e^{-i \lambda} \cos \frac{1}{2} \theta}{2 \sqrt{ } \pi} \sum_{m=0}^{\infty} \frac{(2 i \lambda)^{m}}{m !} \sum_{n=m}^{\infty} \frac{(n-m) ! \sin ^{2 n+1} \frac{1}{2} \theta}{\Gamma\left(n-m+\frac{3}{2}\right)} \\
+ & \frac{e^{-i \lambda} \cos \frac{1}{2} \theta}{2 \sqrt{ } \pi^{3}} \sum_{m=1}^{\infty} \frac{(2 i \lambda)^{m}}{m !} \sum_{n=0}^{m-1} \frac{\Gamma\left(m-n-\frac{1}{2}\right) \sin ^{2 n+1} \frac{1}{2} \theta}{(m-n-1) !} \\
& \quad \times\left\{2 \psi(m-n)-2 \psi(2 m-2 n)+\psi(m+1)+\frac{1}{2} \pi i-\log \frac{1}{2} \lambda\right\} .
\end{aligned}
$$

This interchange needs justification, and the justification follows by proving that the pair of repeated series on the right of the last equation are absolutely convergent. For the former, the series of moduli to be considered is

$$
\begin{aligned}
& \left|\sin \frac{1}{2} \theta\right| \sum_{m=0}^{\infty} \frac{|2 i \lambda| m}{m !} \sum_{n=m}^{\infty} \frac{(n-m) ! \sin ^{2 n} \frac{1}{2} \theta}{\Gamma\left(n-m+\frac{3}{2}\right)} \\
& =\frac{\theta\left|\sin \frac{1}{2} \theta\right|}{\cos \frac{1}{2} \theta \cdot \sqrt{ } \pi} \sum_{m=0}^{\infty} \frac{|2 i \lambda| m \sin ^{2 m-1} \frac{1}{2} \theta}{m !}<\infty
\end{aligned}
$$

provided that $-\pi<\theta<\pi$. For the latter, we use the inequalities

$$
\begin{aligned}
\frac{\Gamma\left(k+\frac{1}{2}\right) \Gamma\left(\frac{1}{2}\right)}{\Gamma(k+1)}=\int_{0}^{1} t^{k-\frac{1}{2}}(1-t)^{-\frac{1}{2}} d t \leqq \pi, & (k=0,1,2, \ldots), \\
|\psi(k+1)| \leqq k+1, & (k=0,1,2, \ldots) ;
\end{aligned}
$$

and the series of moduli to be considered is 


$$
\begin{gathered}
\sum_{m=1}^{\infty} \frac{|2 i \lambda|^{m}}{m !} \sum_{n=0}^{m-1} \Gamma\left(m-n-\frac{1}{2}\right)\left|\sin ^{2 n+1} \frac{1}{2} \theta\right| \\
(m-n-1) ! \\
\quad \leqq \\
\sum_{m=1}^{\infty} \frac{|2 i \lambda|^{m}}{m !}(m \sqrt{ } \pi)\left(2 m+4 m+m(m+1)+\frac{1}{2} \pi i-\log \frac{1}{2} \lambda\right\} \mid \\
<\infty
\end{gathered}
$$

Actually it is clear that we have

$$
\begin{aligned}
F(\lambda, \theta)= & \frac{\theta e^{-i \lambda \cos \theta}}{2 \pi}+\frac{e^{-i \lambda} \cos \frac{1}{2} \theta}{2 \sqrt{\pi^{3}}} \sum_{m=1}^{\infty} \frac{(2 i \lambda)^{m}}{m !} \sum_{n=0}^{m-1} \frac{\Gamma\left(m-n-\frac{1}{2}\right) \sin ^{2 n+1} \frac{1}{2} \theta}{(m-n-1) !} \\
& \times\left\{2 \psi(m-n)-2 \psi(2 m-2 n)+\psi(m+1)+\frac{1}{2} \pi i-\log \frac{1}{2} \lambda\right\},
\end{aligned}
$$

and that the second expression on the right is an integral function of $\theta$ when $\theta$ is regarded as a complex variable, while, if $\lambda$ is regarded as a complex variable, it is a one-valued analytic function of $\lambda$ throughout the $\lambda$-plane when this plane has a cut along the negative half of the real axis.

In particular, we have

$$
F(\lambda, \pi-0)=\frac{1}{2} e^{i \lambda}, \quad F(\lambda,-\pi+0)=-\frac{1}{2} e^{i \lambda} .
$$

For purposes of computation when $\lambda$ is positive and $\theta$ lies between $\pm \pi$, it is probably best to use the expansion

$$
F(\lambda, \theta)=\frac{1}{4} \cos \frac{1}{2} \theta \sum_{n=0}^{\infty} f_{n}(\lambda) \sin ^{2 n+1} \frac{1}{2} \theta
$$

provided that $\theta$ is not very near $\pm \pi$. It is unnecessary to compute all the functions $f_{n}(\lambda)$ which are required by means of the power series; it is sufficient to compute $f_{0}(\lambda)$, to observe that

$$
f_{-1}(\lambda)=\frac{1}{2} \pi\left\{i J_{0}(\lambda)-Y_{0}(\lambda)\right\}
$$

and to use the recurrence formula

$$
(2 n+1) f_{n}(\lambda)=(2 n+4 i \lambda) f_{n-1}(\lambda)-4 i \lambda f_{n-2}(\lambda), \quad(n=1,2,3, \ldots)
$$

in order to compute $f_{1}(\lambda), f_{2}(\lambda), \ldots$ This recurrence formula is easily proved by differentiating

$$
\frac{\sinh \frac{1}{2} t \exp \left(2 i \lambda \cosh ^{2} \frac{1}{2} t\right)}{\cosh ^{2 n+1} \frac{1}{2} t}
$$

with respect to $t$, rearranging the result and then integrating.

We next deal with $G(\lambda, \theta)$ in a similar manner, by starting with the formula

$$
\frac{2}{\pi} \int_{0}^{\infty} \exp \left(2 i \lambda \cosh ^{2} \frac{1}{2} t\right) \cosh \frac{1}{2} t d t=\frac{e^{2 i \lambda+\frac{1}{2 \pi i}}}{\sqrt{ }\left(\frac{1}{2} \pi \lambda\right)}=\frac{e^{\ell \pi i}}{\sqrt{\left(\frac{1}{2} \pi \lambda\right)}} \sum_{m=0}^{\infty} \frac{(2 i \lambda)^{m}}{m !},
$$


and integrating it $n+1$ times from 0 to $\lambda$. The details of the work are somewhat simpler than the corresponding details for $F(\lambda, \theta)$, and we get

$$
\begin{aligned}
(2 i)^{n+1} & {\left[\int_{0}^{\lambda} d \lambda\right]^{n+1} \frac{e^{t \pi i}}{\sqrt{ }\left(\frac{1}{2} \pi \lambda\right)} \sum_{m=0}^{\infty} \frac{(2 i \lambda)^{m}}{m !}=\frac{e^{t \pi i}}{\sqrt{ }\left(\frac{1}{2} \pi \lambda\right)} \sum_{m=0}^{\infty} \frac{\dot{\Gamma}\left(m+\frac{1}{2}\right)(2 i \lambda)^{m+n+1}}{m ! \Gamma\left(m+n+\frac{3}{2}\right)}, } \\
(2 i)^{n+1} & {\left[\int_{0}^{\lambda} d \lambda\right]^{n+1} \frac{2}{\pi} \int_{0}^{\infty} \exp \left(2 i \lambda \cosh ^{2} \frac{1}{2} t\right) \cosh \frac{1}{2} t d t } \\
& =\frac{2}{\pi} \int_{0}^{\infty} \frac{1}{\cosh ^{2 n+1} \frac{1}{2} t}\left\{\exp \left(2 i \lambda \cosh ^{2} \frac{1}{2} t\right)-\sum_{m=0}^{n} \frac{\left.(2 i \lambda)^{m} \cosh ^{2 m} \frac{1}{2} t\right)}{m !} d t\right. \\
& =e^{i \lambda} g_{n}(\lambda)-\frac{2}{\sqrt{ } \pi} \sum_{m=0}^{n} \frac{\Gamma\left(n+\frac{1}{2}-m\right)(2 i \lambda)^{m}}{m !(n-m) !},
\end{aligned}
$$

so that ${ }^{2}$

$g_{n}(\lambda)=\frac{2 e^{-i \lambda}}{\sqrt{ } \pi} \sum_{m=0}^{\prime n} \frac{\Gamma\left(n+\frac{1}{2}-m\right)(2 i \lambda)^{m}}{m !(n-m) !}+\frac{e^{-i \lambda+1 \pi i}}{\sqrt{ }\left(\frac{1}{2} \pi \lambda\right)} \sum_{m=n+1}^{\infty} \frac{\Gamma\left(m-n-\frac{1}{2}\right)(2 i \lambda)^{m}}{(m-n-1) ! \Gamma\left(m+\frac{1}{2}\right)}$

and hence

$$
\begin{aligned}
G(\lambda, \theta)=\frac{e^{-i \lambda} \cos \frac{1}{2} \theta}{2 \sqrt{ } \pi} \sum_{n=0}^{\infty} \sin ^{2 n} \frac{1}{2} \theta & {\left[\sum_{m=0}^{n} \frac{\Gamma\left(n+\frac{1}{2}-m\right)(2 i \lambda)^{m}}{m !(n-m) !}\right.} \\
& \left.+\frac{e^{\ddagger \pi i}}{\sqrt{ }(2 \lambda)} \sum_{m=n+1}^{\infty} \frac{\Gamma\left(m-n-\frac{1}{2}\right)(2 i \lambda)^{m}}{(m-n-1) ! \Gamma\left(m+\frac{1}{2}\right)}\right] .
\end{aligned}
$$

When we rearrange this repeated series, we get

$$
\begin{gathered}
G(\lambda, \theta)=\frac{e^{-i \lambda+\frac{i \pi i}{2} \cos \frac{1}{2} \theta}}{2 \sqrt{ }(2 \pi \lambda)} \sum_{m=1}^{\infty} \frac{(2 i \lambda)^{m}}{\Gamma\left(m+\frac{1}{2}\right)} \sum_{n=0}^{m-1} \frac{\Gamma\left(m-n-\frac{1}{2}\right)}{(m-n-1) !} \sin ^{2 n \frac{1}{2} \theta} \\
+\frac{1}{2} e^{-i \lambda \cos \theta} \operatorname{sgn}\left(\cos \frac{1}{2} \theta\right)
\end{gathered}
$$

so long as $\theta$ is real and not an odd multiple of $\pi$.

This result shows, in particular, that

$$
G(\lambda, \pi-0)=G(\lambda,-\pi+0)=2 e^{i \lambda},
$$

and also that $G(\lambda, \theta)$ defines an analytic function of $\lambda$, regarded as a complex variable, in the $\lambda$-plane cut along the negative half of the real axis, just like $F(\lambda, \theta)$.

In conclusion $I$ remark that it is an easy matter to obtain asymptotic expansions for $f_{n}(\lambda)$ and $g_{n}(\lambda)$ when $\lambda$ is large, in the manner indicated by Copson and Ferrar for $F(\lambda, \theta)$; it also seems to

1 The recurrence formula for $g_{n}(\lambda)$ is

$$
2 n g_{n}(\lambda)=(2 n-1+4 i \lambda) g_{n-1}(\lambda)-4 i \lambda g_{n-2}(\lambda), \quad(n=1,2,3, \ldots \ldots) .
$$


be worth pointing out that, even when $\lambda$ is not smail, the series

$$
\frac{1}{2 i} \sum_{n=1}^{\infty} e^{-t n \pi i} H_{n}^{(1)}(\lambda) \sin n \theta,
$$

which they mention as arising formally in the investigation of $F(\lambda, \theta)$, has its terms ( $q u a$ functions of $n$ ) of such a magnitude that it is completely useless.

The University, Birmingham. 